\title{
Dimensão psicoafetiva do profissional de enfermagem diante do paciente em processo de morte: revisão integrativa
}

\author{
The psychoaffective dimension of the nursing professional in \\ front of the patient in the death process: integrative review
}

Darcília Garcia Arantes' • Emília Cristina de Aguiar Vargas² • Enéas Rangel Teixeira ${ }^{3}$

\begin{abstract}
RESUMO
A morte é um invisível, o cessar de tudo que se amou e desejou presente nos medos, que não se restringe apenas aos pacientes, mas também aos profissionais de saúde, em especial, aos de enfermagem que estão mais próximos do paciente e seus familiares prestando o cuidado em sua integralidade. Portanto, torna-se necessário ampliar discussões sobre a dimensão psicoafetiva do profissional de enfermagem e sua influência no cuidado prestado. 0 estudo tem como objetivo analisar a dimensão psicoafetiva do profissional de enfermagem diante do paciente em processo de morte. Utilizou-se o método de revisão integrativa de literatura. Doze artigos atenderam a questão norteadora da pesquisa e a partir da abordagem destes, foram elencadas três categorias: cuidado de enfermagem ao paciente em processo de morte, preparo para lidar com a morte: reflexos da formação acadêmica, dimensão psicoafetiva do profissional de enfermagem: reflexos no cuidado. 0 estudo identificou que a temática da morte causa pavor e muito sofrimento aos profissionais de enfermagem, influenciando negativamente no cuidado prestado ao paciente e seu familiar. Assim, tornase importante a elaboração de estudos, que novos valores e modos de viver sejam construídos, para que morte e vida se tornem indissociáveis às práticas de saúde.
\end{abstract}

Palavras-chave: Morte; Cuidado de Enfermagem; Sentimentos; Atitude Frente à Morte.

\section{ABSTRACT}

Death is an invisible, the cessation of everything loved and desired, present in the fears, which is not restricted only to patients, but also to health professionals, especially those who are closest to the patient and their relatives providing care in its entirety. Therefore, it is necessary to expand discussions about the psycho-affective dimension of the nursing professional and their influence on the care provided. The objective of this study is to analyze the psychoaffective dimension of the nursing professional in front of the patient in the death process. The integrative literature review method was used. Twelve articles addressed the guiding question of the research and from the approach of these, were listed three categories: nursing care to the patient in the process of death, preparation to deal with death: reflections of the academic formation, psychoaffective dimension of the professional nursing: reflexes in care. The study identified that the issue of death causes dread and much suffering to nursing professionals, negatively influencing the care provided to the patient and his relative. Thus, the development of studies is important, new values and ways of living are built, so that death and life become inseparable from health practices.

Keywords: Death; Nursing Care; Emotions; Attitude to Death. Mestrado Profissional em Enfermagem Assistencial da EEAAC/UFF. Niterói (RJ). Brasil. E-mail: emiliavargas@gmail.com.

${ }^{3}$ Enfermeiro, Pós Doutor, Professor e Orientador do Programa de Mestrado Acadêmico e profissional da Escola de Enfermagem Aurora de Afonso Costa - EEAAC, da Universidade Federal Fluminense - UFF. Niterói (RJ). Brasil. E-mail: eneaspsi@gmail.com. 


\section{INTRODUÇÃO}

A problemática da morte é o acontecimento, é o fato sobre o qual a maioria dos seres humanos se recusa a pensar até serem confrontados com o iminente e pessoal problema. A morte: um invisível, um indefinível, o cessar de tudo que se amou, desejou e conquistou em sua trajetória de vida. E por mais que tenhamos que vivê-la em nosso cotidiano, nos causa assombro. Ela precisa viver à sombra de nós, mesmo que a todo tempo esteja nos margeando, coexistindo com a vida. Diante dela o imponderável se apresenta: impotência e finitude, mortes das mortes de muitos momentos que antecedem o fim que definimos como derradeiro.

A morte, apesar de fazer parte do ciclo natural da vida, é vista como inimiga oculta e vergonhosa por ferir a onipotência do homem moderno ${ }^{1}$. Na contemporaneidade, a morte vem ocorrendo principalmente em hospitais, assistida pela equipe de saúde e por todo um arsenal tecnológico,porém,encontram-se profissionais desprovidos de qualquer preparo para lidar com a morte como algo que está por vir. A morte é nesse ambiente, vista como ideia de falência dos órgãos e fracasso do corpo².

No ambiente hospitalar, a morte, a dor e o sofrimento fazem parte do cotidiano dos profissionais de saúde. Estes profissionais, principalmente os de enfermagem, enfrentam a morte diariamente, pois pela sua dinâmica de trabalho, são os que em maior tempo, permanecem em contato com o paciente e seus familiares realizando suas atividades de cuidado. Porém, para que essas atividades cuidadoras tenham êxito, o enfermeiro além de ter que possuir conhecimento das técnicas e da doença em si, precisa também de habilidades para lidar com os sentimentos dos outros e com suas emoções diante do paciente com ou sem possibilidade de cura ${ }^{3-4}$. Muitas vezes, o contato com essas situações gera sofrimento psíquico e estresse, pois interpreta-se que a morte ocorreu devido à falha no trabalho desenvolvido e fracasso pessoal ${ }^{5}$.

Estudo revela que os profissionais que lidam diretamente com a morte, para sobreviver, precisam de uma dose de negação $0^{6}$, de algo que pode ser denominado como ilusão, fé ou esperança de que a morte não os encontrará4.

Como o tema da morte é pouco discutido no ambiente hospitalar, o profissional criou estratégias de colocála em um lugar de exclusão e silêncio. $\mathrm{E}$ como não há um momento para reflexão e discussão, não ocorre o entendimento de que a morte faz parte da existência e que só a partir desse entendimento é que, talvez, o profissional possa oferecer uma assistência de enfermagem de melhor qualidade ao paciente.

É importante que a doença e a possibilidade de morte sejam vistas como experiências humanas, representando um processo contínuo de aprendizado ${ }^{7}$. Criar um modelo de atenção que aproximasse o profissional do paciente, melhoraria os processos de comunicação, de acolhimento, de autonomização e vínculos.
A partir dessas considerações, torna-se imperativo analisar sobre a temática da morte e da dimensão psicoafetiva do profissional de enfermagem, e como esta pode aproximá-lo ou afastá-lo do paciente no processo de morte.

\section{MÉTODO}

Com a finalidade de responder ao objetivo, o presente estudo utilizou como método a revisão integrativa de literatura. A coleta de dados utilizou a metodologia proposta por Mendes, Silveira e Galvão ${ }^{8}$. Foram utilizadas cinco etapas: $1^{\text {a) }}$ Identificação do tema e escolha da questão norteadora; $2^{a}$ ) Estabelecimento de critérios de inclusão e exclusão dos estudos; $3^{a}$ ) Definição de informações à serem extraídas dos estudos selecionados; $4^{a}$ ) Interpretação dos resultados e $5^{\text {a) }}$ Síntese do conhecimento.

A coleta de dados foi realizada através de consultas nas seguintes bases de dados: Base de dados em Enfermagem (BDENF), Literatura Latino-Americana e do Caribe em Ciências da Saúde (LILACS) e Medical Literature Analysis and Retrieval System Online (MEDLINE).

O levantamento dos artigos ocorreu nos meses de setembro e outubro de 2016. Foram utilizados os Descritores em Ciências da Saúde (DeCS): morte, cuidado de enfermagem e, como dimensão psicoafetiva não é descritor, utilizaram-se os descritores sentimentos e atitude frente à morte.

Para a busca avançada utilizou-se o operador boleano $(A N D)$ na seguinte sequência: morte $A N D$ cuidado de enfermagem $A N D$ sentimentos $A N D$ atitude frente à morte. A escolha desse descritor se deu em função de atender o objetivo do estudo. 0 marco temporal estabelecido foi de 2011 a 2016.

Foram incluídos no estudo, artigos que pudessem contemplar a seguinte questão norteadora: como o profissional de enfermagem se relaciona com o paciente em processo de morte? Os artigos são os disponíveis na íntegra nas bases de dados, escritos por enfermeiros e publicados em português. Foram excluídos 137 artigos: teses, monografias, capítulos de livros e dissertações.

Foram selecionados 149 artigos: 55 na MEDLINE, 54 na LILACS e 40 na BDENF. Desse total apenas 72 artigos estavam na íntegra e desses apenas 12 foram incluídos para análise, pois respondiam ao objetivo proposto por essa pesquisa.

A figura 1 apresenta o fluxograma com a estratégia de busca e seleção das produções que compuseram a amostra.

$\mathrm{Na}$ análise de dados, utilizou-se a descrição dos mesmos. Para tal, criou-se um quadro para apresentação da síntese dos artigos incluídos na revisão. E na discussão, os temas mais citados foram categorizados, a fim de respaldar cientificamente os significados dos dados ${ }^{9}$.

Os preceitos éticos para sua realização obedecem à Resolução n 510/2016 do Conselho Nacional de Saúde. 


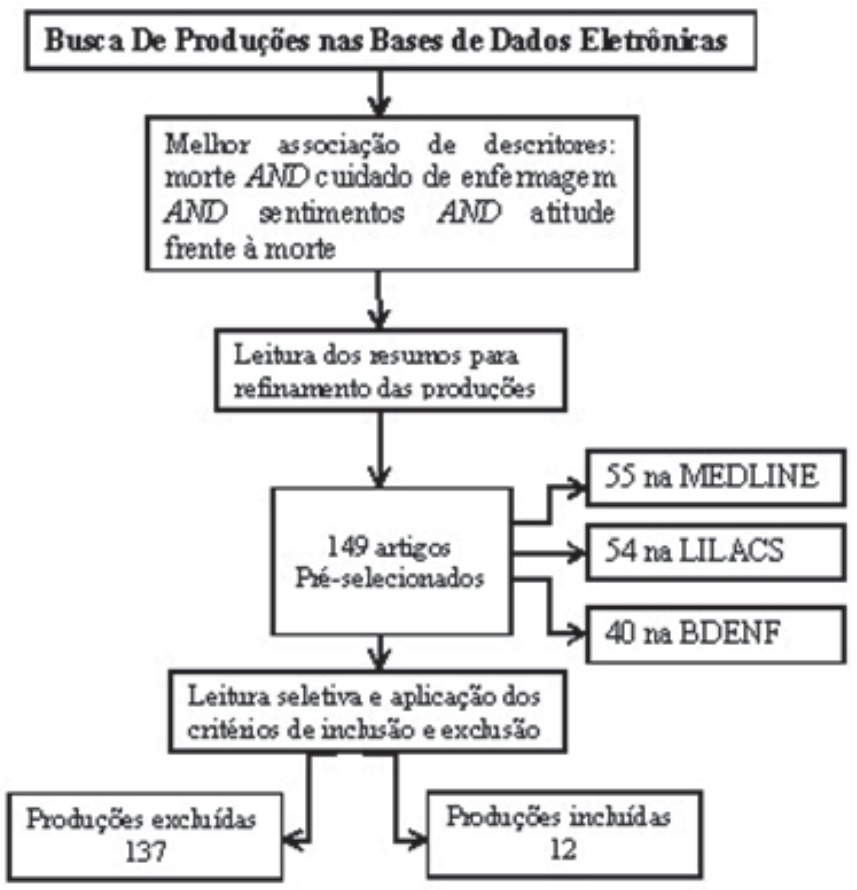

Figura 1: Fluxograma de busca e seleção das produções científicas Fonte: dados da pesquisa.

\section{RESULTADOS}

$\mathrm{Na}$ revisão integrativa foram analisados 12 artigos que atenderam a questão norteadora da pesquisa e que atenderam aos critérios de inclusão e exclusão. Segue abaixo o quadro com os artigos selecionados na íntegra.

Os artigos selecionados foram todos realizados no Brasil. Quanto aos períodos de publicação encontraram-se três de 2011, 2012 e 2016; dois de 2013 e um de 2015.

Com o objetivo de responder a questão da pesquisa, realizou-se a leitura dos artigos na íntegra, buscando identificar a influência da dimensão psicoafetiva do profissional de enfermagem no cuidado ao paciente em processo de morte.

A partir dos temas abordados nos artigos, foram elencadas três categorias: o cuidado de enfermagem ao paciente em processo de morte; (des) preparo para lidar com a morte: reflexos da formação acadêmica; e a dimensão psicoafetiva do profissional de enfermagem: reflexos no cuidado.

Quadro 1: Artigos incluídos na revisão integrativa

\begin{tabular}{|c|c|c|c|c|}
\hline Artigos & Autor & Periódico & Título & Ano \\
\hline $\mathrm{A} 1$ & $\begin{array}{l}\text { Borges MS, } \\
\text { Mendes N. }\end{array}$ & Rev bras enferm & $\begin{array}{l}\text { Representações dos profissionais de saúde sobre a } \\
\text { morte e o morrer }{ }^{10} \text {. }\end{array}$ & 2012 \\
\hline $\mathrm{A} 2$ & $\begin{array}{l}\text { Benedetti GMS, Oliveira K, } \\
\text { Oliveira WT, Sales CA, } \\
\text { Ferreira PC. }\end{array}$ & Rev gaúch enferm & $\begin{array}{l}\text { Significado do processo morte/morrer para os } \\
\text { acadêmicos ingressantes no curso de enfermagem }{ }^{11} \text {. }\end{array}$ & 2013 \\
\hline A3 & $\begin{array}{l}\text { Mota MS, Gomes GC, } \\
\text { Coelho MF, Lunardi Filho WD, } \\
\text { Sousa LD. }\end{array}$ & Rev gaúch enferm & $\begin{array}{c}\text { Reações e sentimentos de profissionais de } \\
\text { enfermagem frente à morte dos pacientes sob os } \\
\text { seus cuidados }{ }^{12} \text {. }\end{array}$ & 2011 \\
\hline A4 & $\begin{array}{l}\text { Barbosa AGC, Massaroni L, } \\
\text { Lima EFA }\end{array}$ & $\begin{array}{l}\text { Rev pesqui cuid } \\
\text { fundam }\end{array}$ & $\begin{array}{c}\text { Significados do processo de morrer e de morte para } \\
\text { a equipe multiprofissional }{ }^{13} \text {. }\end{array}$ & 2016 \\
\hline A5 & $\begin{array}{l}\text { Freitas TLL, Banazeski AC, } \\
\text { Eisele A, Souza EN, } \\
\text { Bitencourt JVO, Souza SS. }\end{array}$ & Enferm glob & $\begin{array}{c}\text { O olhar da enfermagem diante do processo de } \\
\text { morte e morrer de pacientes críticos: uma revisão } \\
\text { integrativa }{ }^{14} \text {. }\end{array}$ & 2016 \\
\hline A6 & $\begin{array}{l}\text { Menossi MJ, Zorzo JCC, } \\
\text { Lima RAG }\end{array}$ & Rev latinoam enferm & $\begin{array}{c}\text { A dialógica vida/morte no cuidado do adolescente } \\
\text { com câncer }{ }^{15} \text {. }\end{array}$ & 2012 \\
\hline A7 & Medeiros YKF, Bonfada D. & Rev RENE & $\begin{array}{l}\text { Refletindo sobre a finitude: um enfoque na } \\
\text { assistência de enfermagem frente à terminalidade }{ }^{16} \text {. }\end{array}$ & 2012 \\
\hline A8 & $\begin{array}{l}\text { Moura KS, Araújo LM, } \\
\text { Araújo LM, Valença CN, } \\
\text { Germano RM. }\end{array}$ & Rev RENE & $\begin{array}{l}\text { A vivência do enfermeiro em terapia intensiva: } \\
\text { estudo fenomenológico }{ }^{17} \text {. }\end{array}$ & 2011 \\
\hline A9 & $\begin{array}{l}\text { Silva RS, Campos AER, } \\
\text { Pereira A. }\end{array}$ & Rev Esc Enferm USP & $\begin{array}{c}\text { Cuidando do paciente no processo de morte na } \\
\text { Unidade de Terapia Intensiva }{ }^{18} \text {. }\end{array}$ & 2011 \\
\hline $\mathrm{A} 10$ & $\begin{array}{l}\text { Scarton J, Poli G, Kolankiewicz } \\
\text { ACB, Rosanelli CLSP, Carton J, } \\
\text { Poli AG. }\end{array}$ & $\begin{array}{l}\text { Rev enferm UFPE on } \\
\text { line }\end{array}$ & $\begin{array}{l}\text { Enfermagem: a morte e o morrer em unidades de } \\
\text { terapia intensiva pediátrica e neonatal }{ }^{19} \text {. }\end{array}$ & 2013 \\
\hline A11 & $\begin{array}{l}\text { Britto SMC, Ramos RS, } \\
\text { Santos El, Veloso OS, Silva AM, } \\
\text { Mariz RGA. }\end{array}$ & Rev cuid & $\begin{array}{l}\text { Representação social dos enfermeiros sobre cuidados } \\
\text { paliativos }{ }^{20} \text {. }\end{array}$ & 2015 \\
\hline A12 & Mariano PP, Carreira L. & $\begin{array}{l}\text { Esc Anna Nery Rev } \\
\text { Enferm }\end{array}$ & $\begin{array}{l}\text { Prazer e sofrimento no cuidado ao idoso em } \\
\text { instituição de longa permanência: percepção dos } \\
\text { trabalhadores de enfermagem }{ }^{21} \text {. }\end{array}$ & 2016 \\
\hline
\end{tabular}




\section{DISCUSSÃO}

Nesta seção serão discutidos os assuntos que predominaram nos artigos selecionados conforme os critérios de inclusão e exclusão. Foram divididos em três categorias, com o objetivo de facilitar à dinâmica, compreensão e problematização das ideias deste estudo.

\section{Categoria 1: Cuidado de enfermagem ao paciente em processo de morte}

Evidenciou-se neste estudo que 05 dos artigos (A1, A5, A8, A9 e A11) problematizam o processo de cuidado. Seguem as discussões pertinentes as ideias desses autores.

Como a formação acadêmica é voltada para a cura, percebe-se que o profissional presta um cuidado técnico que o afasta do paciente. Os cuidados técnicos como higiene, hidratação, e medicação tornam-se rotineiros e iguais, mantendo-se por vários dias sem alterações. Nesse cuidar contínuo há a predominância de um cuidado mecanicista ${ }^{11}$.

0 avanço tecnológico desencadeou a medicalização ${ }^{22}$ da morte associada à cuidados de alta complexidade em pacientes que necessitam deste aparato tecnológico para manter suas funções vitais. No entanto, essa tecnologia prolonga o seu sofrimento e não contribui para o seu processo de morrer ${ }^{5}$.

Outro ponto destacado é que no cotidiano da assistência de enfermagem, o paciente em processo de morte não recebe nenhuma prioridade na assistência, ou quando recebe não é direcionada à sua condição humana, mas sim em função da complexidade do aparato tecnológico que o paciente está usando ${ }^{20}$.

$\mathrm{Na}$ contramão de toda essa mecanicidade encontrase o cuidado paliativo, modelo que transcende o modelo assistencial tradicional, pois é pautado na abordagem holística, interdisciplinar, humanizada e sem intervenções para antecipar ou adiar a morte. É uma modalidade terapêutica que busca resgatar a humanização perdida nas modernas ações de saúde, valorizando a cultura, espiritualidade, valores e crenças ${ }^{10-11,13}$.

O suporte emocional para o paciente e sua família é muito importante, bem como proporcionar um ambiente agradável com a presença da família, uma assistência humanizada e dignidade no processo de morrer ${ }^{11}$.

Outro aspecto de grande relevância para o cuidado é a comunicação entre os profissionais, pacientes e familiares durante o processo de cuidar ${ }^{24}$. As informações devem ser claras e realistas, porém solidárias. É através da comunicação que as necessidades individuais são reveladas e que desperta o sentimento de confiança e vínculo ${ }^{10}$.

$O$ cuidado ao paciente em processo de morte representa um grande desafio para a equipe de enfermagem, pois é preciso reconhecer que mesmo que não haja mais a possibilidade de cura, o cuidado não deve ser negligenciado ${ }^{25}$. E quando a morte for inevitável, medidas devem ser tomadas para que essa morte seja vivenciada com dignidade ${ }^{26}$.

Diante do exposto, torna-se necessário o entendimento que a morte faz parte da experiência de todo ser vivo e talvez assim, o profissional possa realizar a ação cuidadora com mais afeto e menos mecanicidade, proporcionando ao paciente uma morte com paz e o mínimo de angústia.

\section{Categoria 2: (Des) Preparo para lidar com a morte: reflexos da formação acadêmica}

Nesta categoria, os artigos A1, A2, A5, A9 e A10 problematizam as dificuldades encontradas pelos enfermeiros ao cuidar do paciente em processo de morte.

A morte é um fenômeno que pode ser considerado por alguns como perda, ruptura, desintegração, mas também como a grande viagem, descanso, alívio ${ }^{31}$, enfim, um fenômeno que intriga e causa medo. Sendo assim, é necessário que os futuros profissionais recebam preparo durante a sua formação acadêmica para enfrentarem esse evento em seu cotidiano profissional.

Como o processo de formação acadêmica é voltado para a cura, pois é nela que está a maior gratificação do profissional, percebe-se uma lacuna nos currículos ${ }^{27}$. Quando o profissional, no cotidiano do trabalho, precisa lidar com a morte em geral, sente-se despreparado e tende a afastar-se dela. A graduação não oferece conteúdo suficiente para o cuidado ao paciente em processo de morte $\mathrm{e}^{14-18}$.

Durante o processo de formação acadêmica, o profissional de saúde não recebe treinamento e informação sobre o processo de morrer e a morte. A morte não é discutida na graduação ${ }^{10}$. Estudá-la a partir da graduação pode ser uma boa estratégia para lidar com a sua frequente presença, diminuindo assim, o estresse e ansiedade causados por esse assunto ${ }^{11}$.

A partir dessas considerações evidencia-se a relevância de se debater de forma crítica e reflexiva o tema na graduação, além da necessidade de uma revisão curricular dos cursos e da criação de grupos de discussão sobre o tema, para que os profissionais sejam capazes de atuar, nos diferentes cenários hospitalares, sem que sejam aterrorizados pela presença do processo de morrer e morte ${ }^{14-19}$.

\section{Categoria 3: Dimensão psicoafetiva do profissional de enfermagem: reflexos no cuidado}

Dos artigos selecionados, com exceção do $\mathrm{A} 12$, todos expressam sentimentos que de alguma forma despotencializam 0 profissional de enfermagem ${ }^{28}$. Este, diante da morte de um paciente, vivencia vários sentimentos como tristeza, fracasso, impotência, culpa, insegurança, frustração que geram sofrimento e atitude defensiva interferindo no processo de cuidado ${ }^{10-13}$.

Ao enfrentar situações de morte iminente, os profissionais utilizam mecanismos de defesa ${ }^{29}$ como a 
negação e a evasão, evitando falar sobre o assunto. No entanto, ao utilizar tais mecanismos, o profissional cria uma armadura protetora que aparentemente se manifesta como frieza e insensibilidade, influenciando de forma negativa o processo de cuidar ${ }^{12,16-17}$.

Diante de tanto sofrimento, os profissionais não se permitem entrar em contato com suas emoções. Vivenciar a morte do outro nos remete a nossa própria finitude ${ }^{30}$. Mesmo com experiência, cada um reagirá de forma singular. Apresentar dificuldades em lidar com esses sentimentos poderá influenciar de forma não desejável à assistência prestada, sua vida pessoal e afetiva ${ }^{14}$.

Os sentimentos e percepções que afloram nos profissionais diante da morte de um paciente que esteve sob os seus cuidados, são vividos mais intensamente quando há formação de vínculo afetivo entre o paciente e eles, onde ocorre a troca de afetos, responsabilidades, carinho e cuidado. Quando o vinculo é quebrado pelo evento morte, o profissional sofre, afetando sua integridade e seu estado mental. Pois lidar com a morte é angustiante, além de causar sofrimento nos familiares também se reflete no profissional causando um sentimento de profundo pesar ${ }^{10,19-20}$.

Alguns profissionais consideram o preparo do corpo como uma punição pela morte do paciente que não conseguiram salvar. Para outros, o preparo do corpo parece amenizar o sofrimento, pois, é uma forma de cuidar, mesmo que o paciente já não necessite mais ser cuidado ${ }^{12}$.

Diante dessa realidade de tanto sofrimento, alguns profissionais utilizam a religiosidade como aliada para aceitação e enfrentamento da morte, pois conforta e dá esperança. Nos momentos em que o quadro clínico do paciente se agrava, se apegam à religião, buscando na espiritualidade e crenças religiosas, subsídios para o alivio do sofrimento dos pacientes e indiretamente os seus próprios ${ }^{11,15-16}$.

Os sentimentos de orgulho e utilidade são citados quando os profissionais relatam suas contribuições para o conforto e alívio da dor. $\mathrm{O}$ ato de cuidar, de quem está frágil e dependente, possibilita aos profissionais de enfermagem experiências de sentimentos muito agradáveis, sobretudo o de estar sendo útil, o que consequentemente confere-lhes a sensação de desenvolvimento de uma tarefa socialmente nobre.

O trabalho quando funciona como fonte de prazer, seja pela construção da identidade do sujeito, pelo seu reconhecimento e pela realização profissional, permite que o trabalhador se torne sujeito da ação, dominando o trabalho e não sendo dominado por ele ${ }^{21}$.

É necessário que o profissional elabore significados para sua própria morte e a do próximo, com o objetivo de subsidiar a conduta diante dela, possibilitando uma atuação adequada junto aos pacientes e seus familiares ${ }^{13}$.

\section{CONCLUSÃO}

Esta pesquisa identificou que a morte causa um grande temor aos profissionais de enfermagem. Além de proporcionar sentimentos de impotência, fracasso, culpa, insensibilidade e frieza, que influenciam negativamente na qualidade do cuidado oferecido ao paciente e seus familiares.

Observa-se pelos estudos analisados que a formação acadêmica necessita de um olhar mais cuidadoso para a temática, promovendo nos espaços de formação discussões e reflexões para que esse aluno, ao ingressar no campo de trabalho, possa buscar estratégias que o auxiliem a lidar com o processo de morte de uma forma menos sofrida, proporcionando ao paciente um cuidado ético e estético.

A partir dessas considerações, torna-se relevante a discussão de forma crítica e ética do tema nos cursos de graduação, além da necessidade de uma revisão curricular e da criação de grupos de discussão sobre o tema, para que os profissionais sejam capazes de atuar nos diferentes cenários de prática encarando a morte não somente com tristeza e como fracasso, mas também como alívio, parte de um processo natural da vida.

A morte é um processo que pode ser considerado por alguns como finalização, corte, desestruturação gerando um sentimento de impotência diante de forças externas, mas também poderia ser vista como a grande aventura final da vida. Enfim, ao contrario de um fenômeno que intriga e causa medo, pode ser interpretada como uma continuação da vida num outro patamar assim como ocorreu na história de muitas civilizações antigas onde se sofria muito menos por processos naturais como este. Sendo assim, reafirmamos que é fundamental que os futuros profissionais recebam preparo durante a sua formação acadêmica para enfrentarem esse evento em seu cotidiano profissional.

\section{REFERÊNCIAS}

1. Ariès P. A história da morte no ocidente. Rio de Janeiro: Francisco Alves; 1977.

2. Pitta A. hospital, dor e morte como ofício. São Paulo: Hucitec; 1994.

3. Sousa DM, Soares EO, Costa KMS, Pacífico ALC, Parente ACM. A vivência da enfermeira no processo de morte e morrer dos pacientes oncológicos. Texto \& contexto enferm [Internet]. 2009 jan-mar [acesso em 15 jun 2016]; 18(1): 41-7. Disponível em: http://www.scielo.br/pdf/tce/v18n1/v18n1a05.

4. Shimizu HE. Como os trabalhadores de enfermagem enfrentam o processo de morrer. Rev bras enferm [Internet]. 2007 maijun [acesso em 20 abr 2016]; 60(3): 257-62. Disponível em: http://www.scielo.br/pdf/reben/v60n3/a02.pdf.

5. Kovács MJ. Sofrimento da equipe de saúde no contexto hospitalar: cuidando do cuidador profissional. Mundo saúde [Internet]. 2010 [acesso em 9 jun 2016]; 34(4): 4209. Disponível em: http://www.saocamilo-sp.br/pdf/mundo_ saude/79/420.pdf.

6. Santos MA,Hormanez M.Atitude frente à morte em profissionais e estudantes de enfermagem: revisão da produção científica na última década. Ciênc. saúde coletiva [Internet]. 2013 [acesso em 5 jul 2016]; 18(9): 2757-68. Disponível em: http:// www.scielo.br/pdf/csc/v18n9/v18n9a31.pdf. 
7. Fontoura EG, Santa Rosa DO. Vivência da tríade trágica nos cuidados de enfermagem à pessoa na finitude da vida. Ciênc cuid saúde [Internet]. 2013 jul-set [acesso em 9 jun 2016]; 12(3): 508-14. Disponível em: http://www.periodicos.uem.br/ ojs/index.php/CiencCuidSaude/article/viewFile/18066/pdf.

8. Mendes KDM, Silveira RCCP, Galvão CM. Revisão integrativa: método de pesquisa para a incorporação de evidências na saúde e na enfermagem. Texto \& contexto enferm [Internet]. 2008 out-dez [acesso em 10 ago 2016]; 17(4): 758-64 Disponível em: http://www.scielo.br/pdf/tce/v17n4/18.pdf.

9. Minayo MCS. O desafio do conhecimento: pesquisa qualitativa em saúde. 12a. ed. São Paulo: Hucitec; 2010.

10. Borges MS, Mendes N. Representações de profissionais de saúde sobre a morte e o processo de morrer. Rev bras enferm [Internet]. mar-abr 2012 [acesso em 10 ago 2016]; 65(2): 32431. Disponível em: http://www.scielo.br/scielo.php?script=sci_ arttext\&pid=S0034-71672012000200019.

11. Benedetti GMS, Oliveira K, Oliveira WT, Sales CA, Ferreira PC. Significado do processo morte/morrer para os acadêmicos ingressantes no curso de enfermagem. Rev gaúch enferm [Internet]. 2013 mar [acesso em 15 jun 2016]; 34(1): 173-9. Disponível em: http://www.scielo.br/scielo. php?script=sci_arttext \&pid=S1983-14472013000100022.

12. Mota MS, Gomes GC, Coelho MF Lunardi Filho WD, Sousa LD. Reações e sentimentos de profissionais da enfermagem frente à morte dos pacientes sob seus cuidados. Rev gaúch enferm [Internet]. 2011 mar [acesso em 10 ago 2016]; 32(1): 129-35. Disponível em: http://www.scielo.br/scielo. php?script=sci_arttext $\&$ pid=S1983-14472011000100017.

13. Barbosa AGC, Massaroni L, Lima EFA. Significados do processo do morrer e da morte para a equipe multiprofissional Meaning of the process of dying and death for multiprofessional staff. Rev pesqui cuid fundam [Internet]. $2016 \mathrm{abr}$ [acesso em 27 ago 2016]; 8(2): 4510-7. Disponível em: http://www.seer. unirio.br/index.php/cuidadofundamental/article/view/4849.

14. Freitas TLL, Banazeski AC, Eisele A, Souza EN, Bitencourt JVO, Souza SS. O olhar da enfermagem diante do processo de morte e morrer de pacientes críticos: uma revisão integrativa. Enferm glob [Internet]. 2016 [acesso em 11 dez 2016]; 41: 335-47. Disponível em: http://revistas.um.es/eglobal/article/ viewFile/214601/188591.

15. Menossi MJ, Zorzo JCC, Lima RAG. A dialógica vida/morte no cuidado do adolescente com câncer. Rev latinoam enferm [Internet]. 2012 fev [acesso em 15 jul 2016]; 20(1): 12634. Disponível em: http://www.revistas.usp.br/rlae/article/ view/4487.

16. Medeiros YKF, Bonfada D. Refletindo sobre finitude: um enfoque na assistência de enfermagem frente à terminalidade. Rev RENE [Internet]. 2012 [acesso em 30 jul 2016]; 13(4): 845-52. Disponível em: http://www.redalyc.org/ pdf/3240/324027983014.pdf.

17. Moura KS, Araújo LM, Araújo LM, Valença CN, Germano RM. A vivência do enfermeiro em terapia intensiva: estudo fenomenológico. Rev RENE [Internet]. 2011 abr-jun [acesso em 30 jul 2016]; 12(2): 316-23. Disponível em: http://www. revistarene.ufc.br/vol12n2_pdf/a13v12n2.pdf.

18. Silva RS, Campos AER, Pereira A. Cuidando do paciente no processo de morte na Unidade de Terapia Intensiva. Rev Esc Enferm USP [Internet]. 2011 jun [acesso em 3 set 2016]; 45(3):
738-44. Disponível em: http://www.scielo.br/pdf/reeusp/ v45n3/v45n3a27.pdf.

19. Scarton J, Poli G, Kolankiewicz ACB, Rosanelli CLSP, Scarton J, Poli AG. Enfermagem: a morte e o morrer em unidades de terapia intensiva pediátrica e neonatal. Rev enferm UFPE on line [Internet]. 2013 out [acesso em 24 set 2016]; 7(10): 5929-37. Disponível em: http://www.revista. ufpe.br/revistaenfermagem/index.php/revista/article/ download/3324/7385.

20. Britto SMC, Ramos RS, Santos El, Veloso OS, Silva AM, Mariz RGA. Representação social dos enfermeiros sobre cuidados paliativos. rev cuid [Internet]. 2015 jul [acesso em 24 set 2016]; 6(2): 1062-9. Disponível em: http://www.revistacuidarte.org/ index.php/cuidarte/article/view/170.

21. Mariano PP, Carreira L. Prazer e sofrimento no cuidado ao idoso em instituição de longa permanência: percepção dos trabalhadores de enfermagem. Esc Anna Nery Ver Enferm [Internet]. 2016 [acesso em 3 jul 2016]; 20(4): e20160088. Disponível em: http://www.scielo.br/pdf/ean/v20n4/14148145-ean-20-04-20160088.pdf.

22. Mendonça ALO. Healthcare in the era of science and technology: after all, what is health? Hist cienc saudeManguinhos [Internet]. 2014 [acesso em 24 set 2016]; 21(2): 783-8. Disponível em: http://www.scielo.br/pdf/hcsm/ v21n2/0104-5970-hcsm-21-2-0783.pdf.

23. Boemer MR. A morte e o morrer. 3ed. Ribeirão Preto: Holos;1998.

24. Andrade CG, Costa SFG, Lopes MEL. Cuidados paliativos: a comunicação como estratégia de cuidado para o paciente em fase terminal. Ciênc saúde coletiva [Internet]. 2013 [acesso em 25 fev 2016]; 18(9): 2523-30. Disponível em: http://www. scielo.br/pdf/csc/v18n9/v18n9a06.pdf.

25. Hennemann-Krause L. Ainda que não se possa curar, sempre é possível cuidar. Rev HUPE-UERJ [Internet]. 2012 [acesso em 24 set 2016]; 11(2):1 8-25. Disponível em: http://revista.hupe. uerj.br/detalhe_artigo.asp?id=322.

26. Fontoura EG, Rosa DOS. Vivência da tríade trágica nos cuidados de enfermagem à pessoa na finitude da vida. Ciênc cuid saude [Internet]. 2013 [acesso em jul 2016]; 12(3): 50814. Disponível em: http://www.periodicos.uem.br/ojs/index. php/CiencCuidSaude/article/viewFile/18066/pdf.

27. Bandeira D, Cogo SB, Hildebrandt LM, Badke MR. A morte e o morrer no processo de formação de enfermeiros sob a ótica de docentes de enfermagem. Texto \& contexto enferm [Internet]. 2014 [acesso em 11 jan 2017]; 23(2): 400-7. Disponível em: http://www.scielo.br/pdf/tce/v23n2/pt_01040707-tce-23-02-00400.pdf.

28. Rosa DSS, Couto SA. O enfrentamento emocional do profissional de enfermagem na assistência ao paciente no processo da terminalidade da vida. Revista Enfermagem Contemporânea [Internet]. 2015 [acesso em 12 jan 2017]; 4(1): 92-104. Disponível em: https://www5.bahiana.edu.br/ index.php/enfermagem/article/view/467/438.

29. Kübler-Ross E. Sobre a morte e o morrer: o que os doentes têm para ensinar a médicos, enfermeiras, religiosos e aos seus próprios parentes. Menezes P, tradutor. 7a. ed. São Paulo: Martins Fontes, 1996. Disponível em: https:// psicologianicsaude.files.wordpress.com/2012/11/kc3bcblerross-elisabeth-sobre-a-morte-e-o-morrer.pdf. 
30. Kempfer SS, Carraro TE. Temporalidade: o existir e a perspectiva da finitude para o ser-acadêmico-de-enfermagem ao experienciar a morte. Texto \& contexto enferm [Internet]. 2014 [acesso em set 2016]; 23(3): 728-34. Disponível em: http://www.scielo.br/pdf/tce/v23n3/pt_0104-0707tce-23-03-00728.pdf.

31. Kovács MJ. Morte e desenvolvimento humano. 5a. ed. São Paulo: Casa do Psicólogo; 2010. 